\title{
Elimination of Lymphatic Filariasis in Shandong Province, China, 1957-2015
}

\author{
Xiaodan Huang, Xuli Deng, Jingxuan Kou, Xin Liu, Huaiwei Wang, \\ Peng Cheng, and Maoqing Gong
}

\begin{abstract}
China used to be one of the most heavily endemic countries for lymphatic filariasis (LF) in the world. Bancroftian filariasis, which is caused by the filarioid nematode Wuchereria bancrofti, is the only filariasis in Shandong Province. A total of 864 endemic counties (cities) in 16 provinces/autonomous regions/municipalities with a total population of 330 million people were at risk of infection. Shandong Province was a highly LFendemic area in the 1950s, the epidemiological investigation of LF conducted in 1957 indicated that the disease was endemic in 74 counties and the highest microfilaria rate was up to $26 \%$. There were $\sim 5$ million people in the province infected with LF; among which almost 2.5 million people had lymphedema, elephantiasis, or hydrocele. Through vigorous prevention and scientific research, Shandong Province was the first to propose to treat LF with fortified diethylcarbamazine salt, more than 25 million people in the province had taken the salt, and more than 3 million people took intermittent medication. After more than 50 years of unremitting efforts, Shandong Province basically eliminated LF in 1983 and became the first province in China that successfully eradicated LF in 2004, which has played an important role in accelerating the elimination of filariasis in the country in 2007 and has made tremendous contributions to social and economic development of China. Since 1980, Shandong Province has carried out extensive international cooperation as the WHO Collaborating Center for Lymphatic Filariasis. This article is intended to share the experience in eliminating LF to other parts of the world and improve public health capacity in regions such as Africa and Oceania where the disease is still endemic for interest.
\end{abstract}

Keywords: lymphatic filariasis, Culex pipiens pallens, elimination, Shandong Province, China

\section{Introduction}

$\mathbf{L}$ YMPHATIC FILARIASIS (LF) is a neglected tropical disease caused by nematode worms that live in the lymphatic vessels of humans. It is caused by three species of filarioid worms (Wuchereria bancrofti, Brugia malayi, and Brugia timori) and transmitted by mosquitoes. In 2012, it is estimated that LF was prevalent in over 83 tropical and subtropical countries globally with 1.2 billion people living in endemic areas and about 120 million people infected (Ottesen et al. 1997, World Health Organization 2013). LF is regarded as the second leading cause of global disability. The clinical disfiguring manifestations mainly include lymphedema and hydrocele that cause significant social and economic consequences (World Health Organization 2016, Lenk et al. 2016). In 1997, the World Health Assembly (WHA) passed Resolution 50.29, which called on Member States to eliminate LF as a public health issue globally (Knight 2020, WHA 50.29. 2017). Afterward, in 2000, the World Health Organization (WHO) launched the Global Programme to Eliminate Lymphatic Filariasis (GPELF) with the target to eliminate LF by 2020 (World Health Organization 2010, 2017). In 2012, WHO's neglected tropical disease roadmap also confirmed the target to eliminate filariasis by 2020 . The two parallel goals of the program are (1) transmission interruption through mass drug administration with the combination of albendazole and ivermectin in onchocerciasis coendemic areas, and albendazole and diethylcarbamazine (DEC) elsewhere, and (2) alleviation of suffering through morbidity management and disability prevention in clinical LF patients.

After half a century of efforts, in 2004, Shandong Province took the lead in eradicating LF in China, and its prevention and treatment experience was vigorously promoted throughout the country, greatly speeding up the process of eliminating

Shandong Institute of Parasitic Diseases, Shandong First Medical University \& Shandong Academy of Medical Sciences, Jining, China.

(C) Xiaodan Huang et al. 2020; Published by Mary Ann Liebert, Inc. This Open Access article is distributed under the terms of the Creative Commons Attribution Noncommercial License (http://creativecommons.org/licenses/by-nc/4.0/) which permits any noncommercial use, distribution, and reproduction in any medium, provided the original author(s) and the source are cited. 
filariasis in China. Consequently, China submitted the national report on the elimination of LF to the Fourth Global Congress in 2006. Afterward, the WHO approved China as the first country in the world to declare the elimination of filariasis on May 9, 2007. This article reviews the epidemiology and control strategy for LF in Shandong Province, China, so as to share the experience with other countries where the disease is still endemic.

\section{Baseline Assessment}

A rigorous comprehensive assessment of provincial prevalence of LF was conducted in 1957. The sampling survey was conducted by selecting one to three points from each county, and 1000-1500 residents received examination. It was confirmed that the disease was endemic in 74 counties $(113,65.49 \%)$ of Shandong Province. The distribution of bancroftian filariasis extended from Laoling county in the north $\left(37^{\circ} 48^{\prime} \mathrm{N}\right)$ to Yancheng county in the south $\left(34^{\circ} 22^{\prime} \mathrm{N}\right)$, and from Jiaonan county in the east $\left(120^{\circ} 11^{\prime} \mathrm{E}\right)$ to Dongming county in the west $\left(114^{\circ} 48^{\prime} \mathrm{E}\right)$ (Fig. 1). All the counties reported bancroftian filariasis only. Among the 74 counties (cities, districts) endemic for LF in Shandong Province, 41 were hypoendemic areas (microfilariae $[\mathrm{MF}]$ rate $\leq 5 \%$ ), 19 were mesoendemic areas ( $\mathrm{MF}$ rate $5 \%-\leq 15 \%$ ), and 14 were hyperendemic areas (MF rate $>15 \%$ ) (Fig. 1). It was estimated that there were more than 5 million people $(9.31 \%$,
$53,730,000)$ in the province who were infected with LF; among which almost 2.5 million people $(4.65 \%, 53,730,000)$ had lymphedema, elephantiasis, or hydrocele. The average MF rate of $W$. bancrofti was $7.1 \%$ in endemic areas, with the highest up to 26\% (Cui et al. 1984, Deng and Chen 2002). The hyperendemic areas were mainly distributed in Linyi, Jining, and Zaozhuang cities. Culex pipiens pallens was verified as the predominant vectors transmitting bancroftian filariasis in Shandong Province, China. According to the pretreatment (in the early 1950s), the infection rate of mosquito vector in hyperendemic areas was hypothesized to be as high as $37.5 \%$ to $51.9 \%$ (Wang 2005). Generally, the maturation of $\mathrm{MF}$ takes about 12 days. The main transmission season usually last from July to September, during which infected mature infective larvae can be found in mosquitoes.

\section{Control Strategy by Eliminating Infection Sources}

During the 1950-1960s, the combination of vector control and elimination of infection sources was considered to be the most effective way to control LF. Nevertheless, due to the high prevalence of LF in Shandong Province, and because the comprehensive control measures needed enormous time, money, and manpower, it was difficult to put these measures into practice. Since the early 1970s, a total of five to seven large-scale repeated surveys have been conducted in 74 counties across the province, 70 million individual blood

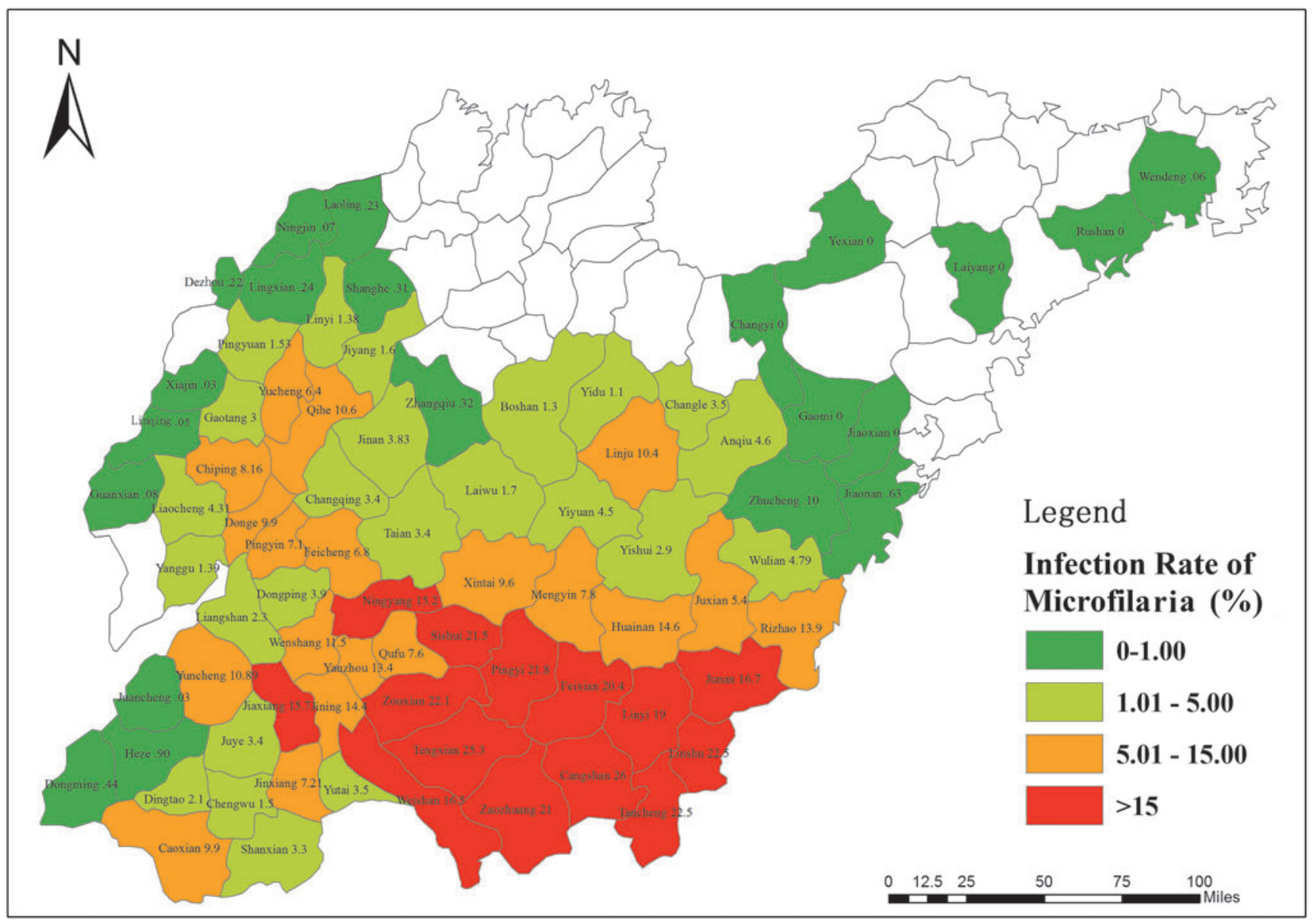

FIG. 1. Epidemiological distribution of 74 counties (cities, districts) endemic for lymphatic filariasis in Shandong Province, China, in 1957. Fig. 1 can be viewed in great detail online. 
samples were taken and 2,943,596 (4.21\%) were found to be positive. All patients were given regular DEC treatment regimen: total dose of $4.2 \mathrm{~g}$ for 7 days, with two to three times at 1-month intervals. The rate of negative conversion was above 95\%. Until 1983, the MF rate in 41 hypoendemic counties (cities, districts) fell to 0.09\% (Zhong and Zhen 1991).

For the counties and cities where the MF rate remained high ( $\mathrm{MF}$ rate $>15 \%$ ), chemotherapy for all the populations and DEC fortified salt was adopted. Antiepidemic and sanitation stations at prefecture and county levels were responsible for collecting blood samples, delivering drugs, and providing DEC salt and other activities. Since 1970, for the 14 counties (cities) residents where the MF rate was between $1 \%$ and $3 \%$, one course of treatment was given. The number of people taking the salt reached 2.03 million, and the MF prevalence dropped to 0.17 . In 1973, among 33 counties (cities) such as Tengzhou and Cangshan, where the MF rate was $\sim 5 \%$, all the populations took DEC fortified salt for 4-6 months. The number of people taking the salt was up to 23.22 million, and the $\mathrm{MF}$ rate was reduced to $0.15 \%$. In addition, a 5-year on-site comparative trial found that the effect of the control strategy by eliminating sources of infection was similar to the strategy by the combination of vector control and elimination of infection sources, there was no significant difference in statistical analysis (Chen et al. 1990, Li et al. 1990). Therefore, the control strategy by eliminating sources of infection was established as the main strategy to control LF nationwide during the 1970s and significantly facilitated the LF control process in China (World Health Organization 1994, Liu and Chen 2004).

\section{Basic Elimination of LF and the Assessment}

In October 1983, the Ministry of Health organized experts to assess the effects of LF control in Shandong Province. Altogether 6 counties (cities), 20 townships (towns), 52 administrative villages were evaluated and 52,898 people received blood tests, only 6 cases of microfilaremia were found, the MF rate was $0.1 \%$ (Sun 1984). It was confirmed that Shandong Province was the first in the country to meet the standard for the basic elimination of LF.

\section{Surveillance After Basic Elimination of LF}

After the basic elimination of LF in Shandong Province, the MF rate and density of endemic areas decreased to extremely low levels. In the following 21 years, according to the law of filariasis transmission and its biological characteristics, monitoring work has been carried out. In 2004, Shandong Province passed the evaluation by the National Expert Group, confirming that Shandong Province has reached the Ministry-issued standards for the elimination of filariasis. The standards included the epidemiological surveillance covered more than $3 \%$ of the total population of the epidemic area and more than $30 \%$ of the popular townships, and no microfilaremia cases were found; mosquito vector monitoring showed no human larval infection (>3000 mosquitoes).

\section{Cross-Sectional Surveillance}

Surveillance was conducted among administrative villages and night thick blood smears were carried out according to the prevalence of LF and the geographical locations of villages. The purpose was to observe the dynamic changes of MF rate from multiple cross-sections so as to lay a foundation for the eradication of the disease. The microfilaremia patients found in the surveillance should be treated with DEC. Cross-sectional monitoring was performed in 74 endemic counties (cities) from 1975 to 1994, a total of 4,323,280 persons were blood examined, and 3783 cases of residual microfilaremia were found. The MF rate gradually decreased from $0.242 \%$ in 1975 to $0.017 \%$ in 1986 (Shi and Sun 1999). No microfilaremia case was detected from 1987 (Fig. 2).

\section{Vector Surveillance}

According to the prevalence of LF, 280 villages in 45 counties (cities) were selected as monitoring points, and about 50 households were selected for each point. Culex pipiens pallens were captured and then dissected and microscopically examined. From 1979 to 2002, mosquito vector

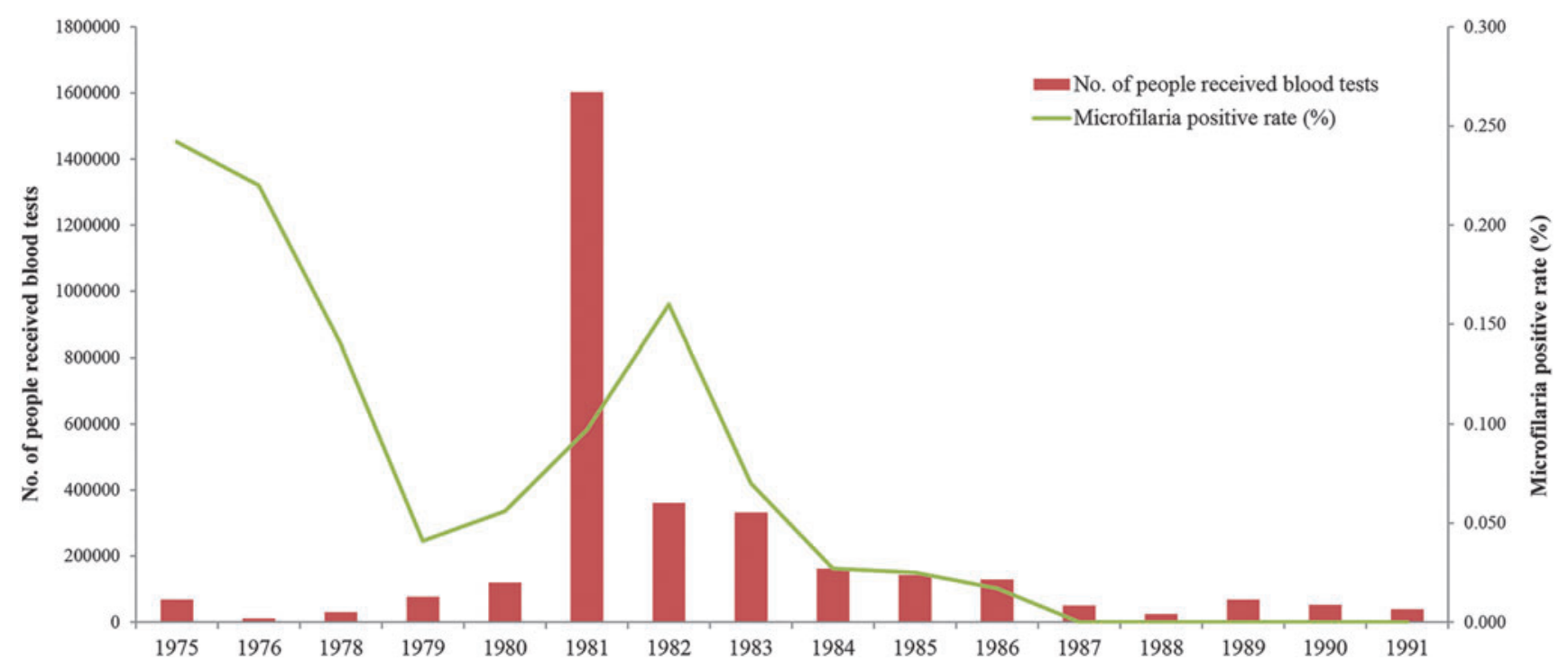

FIG. 2. Number of persons received blood examination and MF rate in cross-sectional monitoring performed in 74 endemic counties (cities) of Shandong Province from 1975 to 1991. 
study was conducted and 721,473 mosquitoes were dissected in 549 villages of 70 counties and 52 positive filarial larvae mosquitoes were found. The average infection rate was $0.007 \%$. In 1988 , more than 279,000 Culex pipiens pallens were dissected and no positive mosquitoes were found (Liu et al. 1988, Gao et al. 1998) (Fig. 3).

\section{Serological Surveillance}

Surveillance sites were established in 13 cities based on the geographic locations, control measures, and prevalence of LF. The blood was collected from the earlobe of $>1$-year-old patients, and the antibody was detected by indirect immunoinfluorescence assay (IFA), and the specific IgG4 level was detected by enzyme-linked immunosorbent assay (ELISA). A total of 10,667 people were tested by IFA, and 148 were positive for antibody with an average positive rate of $1.39 \%$, which had reached the level of healthy people in nonendemic areas (Gao et al. 1994). IgG4 level was tested among 3854 people by ELISA, 11 were found positive and the IgG4-positive rate was $0.29 \%$. No microfilaremia patients were found after the reexamination of the blood in 2000 (Chen et al. 2002).

\section{Floating Population Surveillance}

The surveillance was conducted in areas with frequent population movements. For people from infected areas or staying at the infected areas for more than half a year, blood or serological tests were supposed to be performed to assess the impact of floating populations on filariasis elimination program. Microfilaremia cases should receive regular treatment. From 1984 to 1992, floating population monitoring work was conducted in 19 counties (cities) of Shandong Province. A total of 265,596 persons received blood examination and 147 microfilaremia cases that mainly came from Anhui, Jiangsu, and Sichuan provinces were observed, and no microfilaria cases were found in floating population after 1993 (Fu et al. 2006).

\section{Longitudinal Surveillance}

After the basic elimination of filariasis in 1983, longitudinal surveillance sites were conducted in Shandong Province. A total of 18 villages were selected as monitoring points, including 4 villages in Cangshan County (original MF rate 26.0\%), 3 villages in Tancheng County (original MF rate $22.5 \%$ ), 4 villages in Yicheng District (original MF rate $21.0 \%$ ), 3 villages in Jiaxiang County (original MF rate $15.7 \%$ ), and 4 villages in Ningyang County (original MF rate $15.2 \%$ ). After the prevention and treatment, the MF rate had decreased to $0.29 \%, 0.48 \%, 0.14 \%, 0.10 \%$, and $0.5 \%$, respectively by 1980 . A total of 80,731 persons were examined and 32 cases of residual microfilaremia cases were found. All residual microfilaremia cases became negative between 1986 and 1989 after the control measures were terminated. About 382,549 Culex pipiens pallens were identified and 157 were found positive for filarial larvae. Since 1988, except for three mosquitoes with stage I larvae in 1991, no positive mosquitoes were found. In additon, immunological detection methods that use a monoclonal antibody (McAb)-ELISA for the detection of filarial-specific IgG4 was studied in Shandong Province. In 1995, 760 filter paper samples were tested, and the positive rate of filariasis-specific IgG4 antibody was 0.79 . No microfilaria was found after reexamination (Cui et al. 1989, Sheng et al. 1995). This diagnostic method has been listed as the national filariasis diagnostic standard (WS260-2006).

\section{Investigation and Community Care for Patients with Chronic Filariasis}

After the basic elimination of LF, a total of 1,219,619 people were investigated in 13,489 villages of 50 counties (cities) from 1983 to 1992 . The investigation found that 11,207 patients had symptomatic signs, with an average prevalence of 0.91. There were few new cases of elephantiasis, but there were still a large number of new cases of chyluria. In 1998, an investigation was conducted again in the epidemic areas, and found that the prevalence of chyluria had dropped to $0.19 \%$, which was 30\% lower than that in 1985 (Gu et al. 1999).

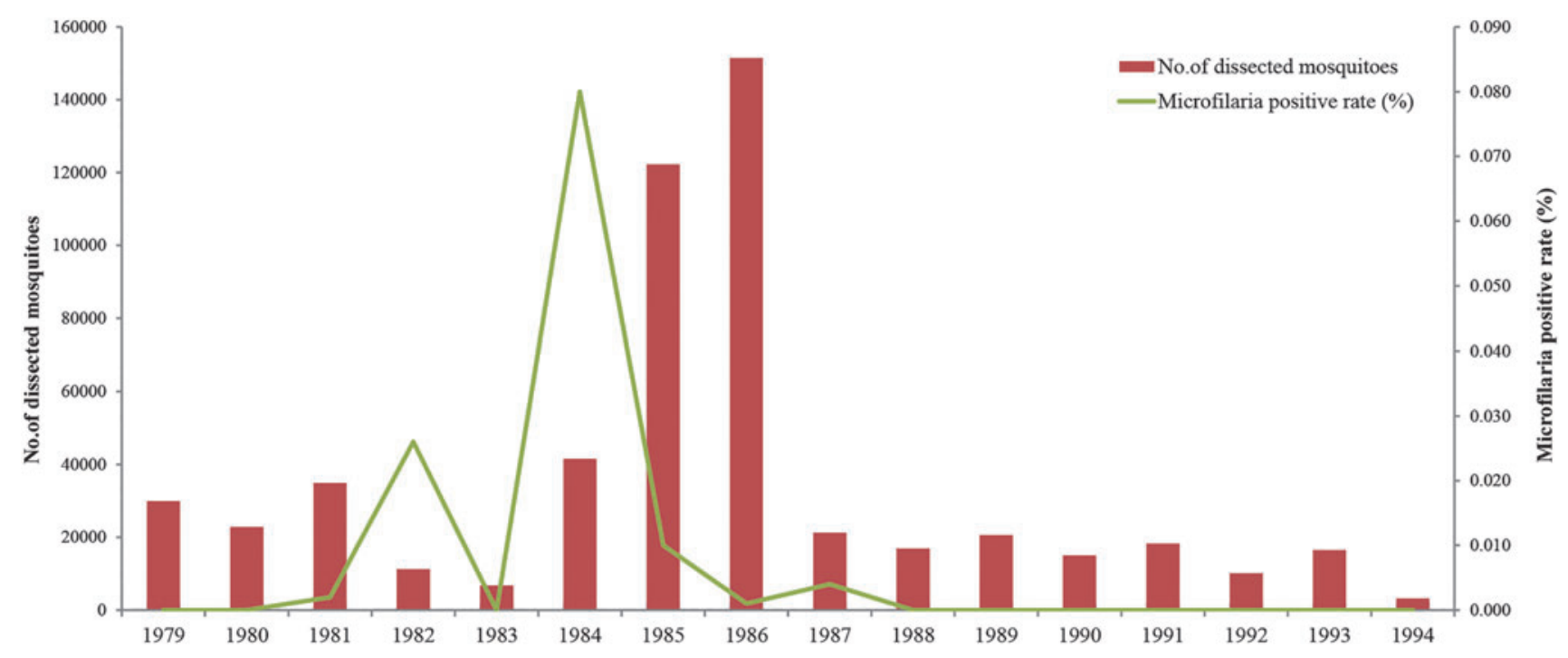

FIG. 3. Number of mosquitoes dissected and MF rate in the cross-sectional monitoring performed in 70 endemic counties in Shandong Province from 1979 to 1994. 
Traditional Chinese medicine, physical therapy, and surgery have been used to treat symptoms of chronic filariasis. For example, audio electrotherapy combined with binding of the affected limb was applied to treat elephantiasis in the lower limbs. Audio electrotherapy has a significant softening effect on scar tissue. The binding of the affected limb produces a certain amount of compression, which can indirectly promote the return of lymph fluid, thereby consolidating the effect of audio electrotherapy (Qiu and Hou 1980). A series of Chinese herbal medicine were identified as the therapeutic agents for chronic lymphedema, lymphangitis, and chyluria patients. The effective herbal medicine antitumor chylomicron preparation was developed. Patients with chyluria were given $12 \mathrm{~g} /$ day for three times per day. The basic cure rate after one course of treatment (usually 3 months) was $90.7 \%$. Patients with limb elephantiasis were treated for three to four courses and the effective rate was $65.7-73.9 \%$ (Wang et al. 1989). To date, the vast majority of patients have been treated effectively.

After the elimination of LF in Shandong Province, community care and symptomatic treatment for patients with chronic filariasis was mainly carried out through (1) investigation and filing; (2) setting up care centers for patients with chronic filariasis; and (3) carrying out health education and other ways of community care to actively alleviate the pain of the disease and improve their quality of life. Up to 2015, more than 3000 people with chronic symptoms have been effectively treated, of whom 486 people were hospitalized (Wang et al. 2016).

\section{Transmission Threshold of Bancroftian Filariasis}

In 1982, the MOH authorized to take research on the transmission threshold of filariasis as a priority project. Tancheng County, a hyperendemic area was selected for the study in Shandong Province from 1984 to 1993. Blood tests on residual mf patients were carried out every 2 years without taking any preventive measures. The relationship between larval infection rate and human microfilaria rates was monitored. The biting density of mosquito and the usage of mosquito nets were investigated during the transmission seasons. The results were as follows: (1) the MF rate in the first 3 years were $0.56-0.64 \%$ and decreased to $0.12 \%$ in 1993 . (2) Among the nine residual microfilaremia cases, eight cases turned negative during the second to the tenth year. (3) The mosquito infection rate of filarial larvae was $0.21 \%, 0.19 \%$, and $0.06 \%$ in the first 3 years, respectively. In 1993, no filarial larvae were found in mosquitoes. (4) The man-biting density of mosquito vectors was 24.1-52.5 mosquitoes/person/night, and 13.5-21.0 mosquitoes/person/ night with bed-nets. Combined with the parameters of vector entomology and etiology, the transmission threshold of bancroftian filariasis was confirmed as follows: the microfilaria rate was $\leq 1$ and the average density of microfilariae was 3-10/ $60 \mu \mathrm{L}$ blood (Shi 1994, Wang et al. 1997). That is, when the population microfilaria rate was $<1 \%$, even if the mosquito density was relatively high, LF was not capable to spread in a large area. This provided a scientific basis for the country to develop standards for the elimination of LF.

\section{Study on Diagnosis and Monitoring Methods of LF}

Study on application of McAb-ELISA to filaria surveillance by detecting that filaria-specific IgG4 was carried out in Shandong Province. In this study, strains of filaria-specific
$\mathrm{McAb}$ were established through cellular fusion and biochemistry-immunology, and were used in filarial infection test as probes to detect filaria-specific IgG4. The positive rate of specific IgG4 in the serum by ELISA was $95.91 \%$ in a group of mf carriers; this was reduced to $1.45 \%$ after treatment. No cross action were tested with serums collected from residents of nonendemic area, including those infected with intestinal nematodes, liver fluke, and cysticercosis. Its specificity was $100 \%$ (Xu et al. 1996, Chen et al. 2002). It was also feasible and practical when applied to field study, effectively reflected the epidemic situation of local filarial infection.

\section{Discussion}

After more than half a century's scientific prevention and unremitting efforts, LF, which seriously endangered the health of the people, has been completely eliminated in Shandong Province, China. The main characteristics and innovations were as follows: (1) the first in the world that established an integrated prevention and control strategy based on the elimination of infectious sources; (2) the first in the world that formulated large-scale chemotherapy with DEC, especially the fortified DEC salt. After application, significant control effects were achieved. This breakthrough has laid a good foundation for the elimination of LF in the country and the world; (3) revealed the transmission law in the later stage of prevention and control, explored the transmission threshold, and found out the indications for interrupting the transmission of LF; (4) established and improved integrated surveillance system, guiding the province to achieve the goal of interrupting transmission, and eliminating filariasis in a planned and phased manner; (5) explored the sensitive, simple, and rapid immunological diagnosis method for detecting filariasis-specific IgG4 by McAbELISA. After the promotion and application, detection method has got satisfactory effect.

At present, many countries have confirmed that LF has been eliminated as a public health problem, such as Cambodia, Cook Islands, Egypt, Maldives, Marshall Islands, Niue, Sri Lanka, Thailand, Togo, Tonga, and Vanuatu, etc. (Sudomo et al. 2010, Charlie et al. 2018, Khieu et al. 2018). However, by the end of 2016, preventive chemotherapy is still needed in 52 countries (Chet et al. 2017, Joseph et al. 2018, Obiora et al. 2018). There are still many challenges to eliminate filariasis by 2020 . For example, it remains a heavy disease burden for the government like Bangladesh and the Republic of Congo, where implementation of large-scale drug treatment is required. What's more, some Pacific island countries do not have sufficient experience in preventing recurrence although local transmission has been interrupted.

\section{Conclusion}

Shandong Province is a region with severe filariasis. It has witnessed the entire process of large-scale prevention and treatment of filariasis, and has accumulated comprehensive experience in prevention and control, scientific research, and medical treatment of filariasis. Shandong Institute of Parasitic Diseases (SIPD) has been a collaborating center with the WHO since 1980 and carried out extensive cooperation with other countries.

In the background of openness and the Belt and Road Initiative, more and more technicians and laborers will work in the countries along the Belt and Road. Nonimmune foreigners 
are more likely to be infected when exposed to local pathogens. In addition, there has been an increasing trend of imported parasitic diseases from abroad in China (Wang 2007, Zhou et al. 2016, Zou et al. 2017, Wang et al. 2018). China has always maintained a high degree of vigilance against imported filariasis. In recent years, there have been reports of Loa loa filariasis and onchocerciasis infection among foreign aid workers (Zhang et al. 2018), reminding us that we must always pay attention to the prevalence of imported filariasis patients with possible epidemic hazards. Therefore, it is quite meaningful for countries to cooperate with each other in the field of disease control and prevention. To achieve the goal of elimination of filariasis by 2020, a strengthened strategy may be needed. Therefore, the promotion of Shandong Province's experience in eliminating LF and its cooperation in the field of health care may greatly enhance the multilateral governance capacity and role of regional and global health filariasis diseases in the country.

\section{Author Disclosure Statement}

No conflicting financial interests exist.

\section{Funding Information}

This study was supported by the National Natural Science Foundation of China (no. 81672059 and 81871685), the Key Research and Development Program of Shandong Province (no. 2019GSF111006), Project of Medical and Health Technology Development Program in Shandong Province (no. 2017WS102), and the Innovation Project of Shandong Academy of Medical Sciences.

\section{References}

Charlie A, D Ramaiah K, Eric O. Elimination of lymphatic filariasis as a public health problem from the Cook Islands. Trop Med Health 2018; 461:12.

Chen JT, Cui ZH, Li WB. Comparative studies on integrated measures and case treatment in control of bancroftian filariasis. Papers of Filariasis Control and Research in China. Fuzhou, China: Fujian Publishing House of Science and Technology, 1990:256-260.

Chen XX, Deng XL, Li GP, Xu FQ, et al. Study on application of McAb-ELISA to filaria surveillance by detecting filariaspecific IgG4. Chin Public Health 2002; 18:579-580.

Chet RO, Basant J, Khagendra P, Shyam P, et al. Impact of mass drug administration for elimination of lymphatic filariasis in Nepal. Negl Trop Dis 2017; 11:e0005788.

Cui ZH, Sheng Q, Yang BJ, Lin XJ, et al. Epidemiological surveillance of filariasis after its being basically eradicated in Shandong Province. Chin J Parasitol Parasit Dis 1989; 7:4-7.

Cui ZH, Xu JF, Wang ZS, Li WB, et al. Research on prevention ancontrol of filariasis in Shandong Province in the past ten years. Chin J Parasitol Parasit Dis 1984; 2:66-72.

Deng XL, Chen XX. Advances in research on prevention and treatment of filariasis in Shandong Province. Chin J Parasit Dis 2002 ; 15:82-84.

Eneanya OA, Cano J, Dorigatti I, et al. Environmental suitability for lymphatic filariasis in Nigeria. Parasit Vectors 2018; 11:513.

$\mathrm{Fu} \mathrm{ZY}$, Gui JJ, Miao F. Surveillance and analysis of filariasis in 1984-2002 after elimination of filariasis in Shandong Province. Chin J Vector Biol Control 2006; 17:102-103.
Gao CL, Cao WC, Chen XX. Changes in anti-filarial antibody after control of filariasis in Shandong Province. Chinese Med J 1994; 107:360-364.

Gao CL, Sheng Q, Deng XL, Zhao ZP, et al. Study on the monitoring of filariasis prevention and control in Shandong Province. Paras Dis Infect Dis 1998; 6:65-67.

Gu XT, Sun JL, Yuan CM, Du BY, et al. Longitudinal investigation and analysis of filarial chyluria in Cangshan County, Shandong Province. Chin J Parasitic Dis Control 1999; 12: 309-318.

Joseph BK, Santigie S, Abdul C, Jusufu P, et al. Progress on elimination of lymphatic filariasis in Sierra Leone. Parasit Vectors 2018; 11:334.

Khieu V, Or V, Tep C, Odermatt P, et al. How elimination of lymphatic filariasis as a public health problem in the Kingdom of Cambodia was achieved. Infect Dis Poverty 2018; 7:15.

Knight R. Lymphatic filariasis. In: Oxford Textbook of Medicine (6 edn). Oxford: Oxford University Press, 2020. doi: 10.1093/med/9780198746690.003.0174.

Lenk EJ, Redekop WK, Luyendijk M, Rijnsburger AJ, et al. Productivityloss related to neglected tropical diseases eligible for preventive chemotherapy: A systematic literature review. PLoS Negl Trop Dis 2016; 10:e0004397.

Li XP, Gan YC, Pan SX. Observation on effect of different intervention measures against bancroftian filariasis. Papers of Filariasis Control and Research in China. Fuzhou, China: Fujian Publishing House of Science and Technology, 1990: 254-256.

Liu X, Chen XX. Experience in the control of filariasis in Shandong Province. Chin Trop Med 2004; 4:371-372.

Liu X, Sheng Q, Zhao ZP, Gao CL, et al. Cross-sectional surveillance after basic eradication of Bancroftian Filariasis in Shandong Province. Chin J Public Health 1988; 14:79-80.

Ottesen EA, Duke BO, Karam M, Behbehani K. Strategies and tools for the control/elimination of lymphatic filariasis. Bull World Health Organ 1997; 75:491-503.

Qiu GY, Hou LQ. Observation on the therapeutic effect of audio electrotherapy combined with binding of the affected limb to treat elephantiasis. Shandong Med 1980; 4:32-33.

Sheng Q, Ren ZX, Gao CL, Cao XC, et al. Longitudinal monitoring analysis after 10 years of basic elimination of filariasis in Shandong Province. Chin J Pathogen Biol 1995; 8:87-90.

Shi Z, Sun D. Achievements in the research on filariasis in China in the past 50 years. Chin J Parasitol Parasit Dis 1999; 17:267-270.

Shi ZJ. Study on the transmission threshold of filariasis. Collaborating Research Group on the Transmission Threshold of Filariasis. Chin J Parasitol Parasit Dis 1994; 12:1.

Sudomo M, Chayabejara S, Duong S, Hernandez L, et al. Elimination of lymphatic filariasis in Southeast Asia. Adv Parasitol 2010; 72:205.

Sun DJ. Filariasis prevention and control in China. Chin J Parasitol Parasit Dis 1984; 2:12.

Wang PY. Study on the elimination of lymphatic filiariasis in Shandong Province. Chin Trop Med 2005; 5:1447-1450.

Wang PY, Yin S, Gao CL, Sheng Q, et al. Study on the transmission threshold of Bancroftian Filariasis in Shandong Provinc. Chin J Parasit Dis Control 1997; 11:11-15.

Wang X, Ruan Q, Xu B, Gu J, et al. Human African trypanosomiasis in emigrant returning to China from Gabon, 2017. Emerg Infect Dis 2018; 24:400-404.

Wang YW. A case of blindness caused by onchocerciasis. Chin J Ophthalmol 2007; 17:287. 
Wang ZL, Fu TX, Hu YX, Wei YB, et al. Clinical analysis of filarial chyluria patients in Shandong Province. Paras Dis Infect Dis 2016; 14:231-234.

Wang ZS, Gong MQ, Tian GM, Cao WC, et al. Clinical obsevation on the control of chyluria with Medium carbon triglyceride produced in China. Chin J Pathogen Biol 1989; 2:101-103.

WHA 50.29. Elimination of lymphatic filariasis as a public health problem. Available at: www.who.int/neglected_diseases/ mediacentre/WHA_50.29_Eng.pdf. Accessed November 30, 2017.

World Health Organization. Global Programme to Eliminate Lymphatic Filariasis: Progress report 2000-2009 and strategic plan 2010-2020. Geneva: WHO, 2010.

World Health Organization. Global programme to eliminate lymphatic filariasis: Progress report for 2012. Wkly Epidemiol Rec 2013; 88:389-399.

World Health Organization. Global programme to eliminate lymphatic filariasis: Progress report, 2015. Wkly Epidemiol Rec 2016; 91:73-88.

World Health Organization. Global programme to eliminate lymphatic filariasis: progress report, 2016. Wkly Epidemiol Rec 2017; 92:594-607.

World Health Organization. Lymphatic filariasis infection \& disease: control strategies. TDR/CTD/FIL/Peneng/94.1. Geneva: WHO, 1994.

Xu FQ, Li GP, Li CT, Sheng Q, et al. Detection of specific IgG4 in the surveillance of filariasis. Chin J Parasitic Dis Control 1996; 9:119-122.

Zhang X, Jiang P, Liu RD, Liu SR, et al. Including content on imported parasitic diseasess when teaching medical parasitology. Chin J Pathogen Biol 2018; 13:112-116.
Zhong $\mathrm{CH}$, Zhen TM. Control and surveillance of filariasis in Shandong. Chin Med J 1991; 104:179-185.

Zhou TC, Sun JL, Wu WP, Shi GQ, et al. Investigation on an imported case of Loiasis. Dis Surveill 2016; 31:796-798.

Zou Y, Wang L, Wang F, Li JJ, et al. Imported Loiasis: A retrospective analysis of 11 cases. Chin J Pathogen Biol 2017; $12: 274-277$.

Address correspondence to: Maoqing Gong Shandong Institute of Parasitic Diseases Shandong First Medical University \& Shandong Academy of Medical Sciences Jining 250062 Shandong Province China

E-mail: mqgong@sdfmu.edu.cn

Peng Cheng Shandong Institute of Parasitic Diseases Shandong First Medical University \& Shandong Academy of Medical Sciences Jining 250062

Shandong Province China

E-mail: cpzuye@aliyun.com 